\title{
Study of a periodic spectral fluctuation existing in a fibered optical feedback cavity-enhanced absorption spectroscopy (OF-CEAS)
}

\author{
Zhifu Luo(, Zhongqi Tan and Xingwu Long ${ }^{*}$
}

\begin{abstract}
A special periodic spectral fluctuation is observed during the study of a fibered high sensitivity optical feedback cavity-enhanced absorption spectroscopy (OF-CEAS) for the measurement of trace gas. This spectral fluctuation is different from some phenomenon observed in former OF-CEAS which contain a resonant cavity with V-shaped configuration, such as the etalon effect and the spectral ripple effect. To reveal why this phenomenon happens and how it works, a series of hypothesis are proposed and tested, and the results show that the multi-beam interference of resonance light at the input mirror of the resonant cavity is the main reason for this phenomenon. Based on the multibeam interference theory, a mathematical modeling of this phenomenon is built, and the theoretical analyses agree well with the experimental results. Some methods to eliminate this phenomenon are proposed and implemented, and the $1 \sigma$ noise equivalent absorption coefficient of $7.6 \times 10^{-10} \mathrm{~cm}^{-1} \mathrm{~Hz}^{-1 / 2}$ is attained with this robust and compact OFCEAS system.
\end{abstract}

Keywords: Optical feedback, Spectral fluctuation, Cavity-enhanced absorption spectroscopy, Human breath analysis

\section{Introduction}

There is an urgent requirement for fast, high sensitivity, low detection limit and real-time trace-gas detectors for atmospheric sciences, environmental monitoring, agriculture process control and medical diagnostics nowadays $[1,2]$. For example, the multi-component analysis of gas is a significant but hard work in breath gas analysis when concentration of some gas species are large and others are at extremely small, and this is extremely correlative for monitoring the exhaled human breath which contains many kinds of breath molecules at trace level. Ordinary gas analytical techniques for multicomponents analysis are mass spectrometry (MS) or gas chromatography (GC) [3]. However, these techniques require expensive equipment and complicated sample preparation which makes real-time measurement not feasible. Detectors based on high finesse optical resonant

\footnotetext{
* Correspondence: xwlong110@sina.com

Department of Optoelectronic Engineering, College of Advanced Interdisciplinary Studies, National University of Defense Technology, No. 1, Deya Road, Kaifu District, Changsha 410073, Hunan, China
}

cavity can achieve accurate and quantificational tracegas detection with high sensitivity and fast response; in addition, these techniques can provide real-time analysis owing to their noninvasive and simple sample preparation features [4]. Owing to these characteristics, these methods appear to be the most promising in trace gas detection.

When the single-wavelength narrow linewidth semiconductor laser is applied in optical resonant cavity absorption spectroscopy, it seems to be the optimal selection for the detection of small gas molecules which present separated absorption lines. For example, the distributed feedback (DFB) diode laser is commercially available owing to the development needs of optical fiber communication. These compact laser source present a low power, meanwhile they provide a continuous frequency-tuning range which is extremely wider comparing to the full width at half maximum (FWHM) of molecular absorption line. Small gas molecules such as $\mathrm{CO}, \mathrm{CO}_{2}, \mathrm{NH}_{3}, \mathrm{HCN}, \mathrm{C}_{2} \mathrm{H}_{6}$, $\mathrm{H}_{2} \mathrm{O}$, and $\mathrm{H}_{2} \mathrm{~S}$ all show strong and narrow fundamental vibrational transitions in middle-infrared, simultaneously, 
overtone transitions lie in the near-infrared band are one to two orders of magnitude weaker in line strength. However, in the near-infrared spectral region, the DFB semiconductor lasers and photo-detectors are relatively cheaper, and these devices perform higher capability at room temperature compared to the devices working in middle-infrared region.

In the application of laser absorption spectroscopy using high finesse optical resonant cavity, representatively, cavityenhanced absorption spectroscopy (CEAS) and cavity ringdown spectroscopy (CRDS) [5], some undesirable phenomenon often occurs in the absorption spectrum, such as the etalon effect and the spectral ripple effect [6]. These effects usually lead to the decline of system performance and restrict its applications. Therefore, it is very significant to explore why these effects happen and how they work, and find ways to eliminate or weaken their influence on the system performance $[7,8]$. In this paper, a compact and robust OFCEAS setup is presented with some fibered components. Owing to the easy replacement of DFB laser for this fibered setup, different gas molecules can be detected with the same device, as long as the selected wavelengths are in the spectral range of the cavity mirrors. A different periodic spectral fluctuation existing in absorption spectroscopy is observed during the study of this OF-CEAS experimental setup. Therefore, ways to prevent and eliminate this phenomenon is proposed. These works are significant to guide the design of fibered OF-CEAS system with V-shaped cavity and to improve the system performance.

\section{Experimental apparatus}

The minimum detectable absorption coefficient of ordinary spectrometer can be up to $10^{-4} \mathrm{~cm}^{-1}$, while it can be up to $10^{-9} \mathrm{~cm}^{-1}$ for the OF-CEAS and OFCRDS techniques [9]. At first, CEAS was presented by Engeln et al. [10] as an improvement of CRDS in 1998. In principle, CEAS and CRDS have similar operation concept. In both techniques, the base of the experimental system is a high finesse optical resonant cavity which is formed with high reflectivity mirrors, and the high quality cavity generates a long effective optical length which can be thousands of times longer than the cavity length. In this paper, we present a fibered OF-CEAS system, and the apparatus is shown in Fig. 1. In our experimental setup, the optical cavity has a typical V-shaped configuration in which two equal arms $(250 \mathrm{~mm})$ forming a folded angle of $7.4^{\circ}$. The glassceramic is used as the cavity body for its ultralow expansion coefficient $\left(\sim 10^{-8} /{ }^{\circ} \mathrm{C}\right)$, and the cavity mirrors are fabricated with fused quartz (diameter is $25 \mathrm{~mm}$ ). Among them, the incident mirror (M0) is a plane mirror, M1 and M2 are spherical mirrors with $2 \mathrm{~m}$ radius, and the device combining M2 and a piezoelectric ceramic transducer (PZT) is used to adjust the cavity length in a tiny region. The ultra-low loss coating lie in the central area of the mirrors $(R \approx 99.99 \%$ at $1530 \pm 30 \mathrm{~nm}$, diameter is $10 \mathrm{~mm}$ ). Finally, the cavity's main body and three mirrors constitute a steady optical cavity length of $500 \mathrm{~mm}$.

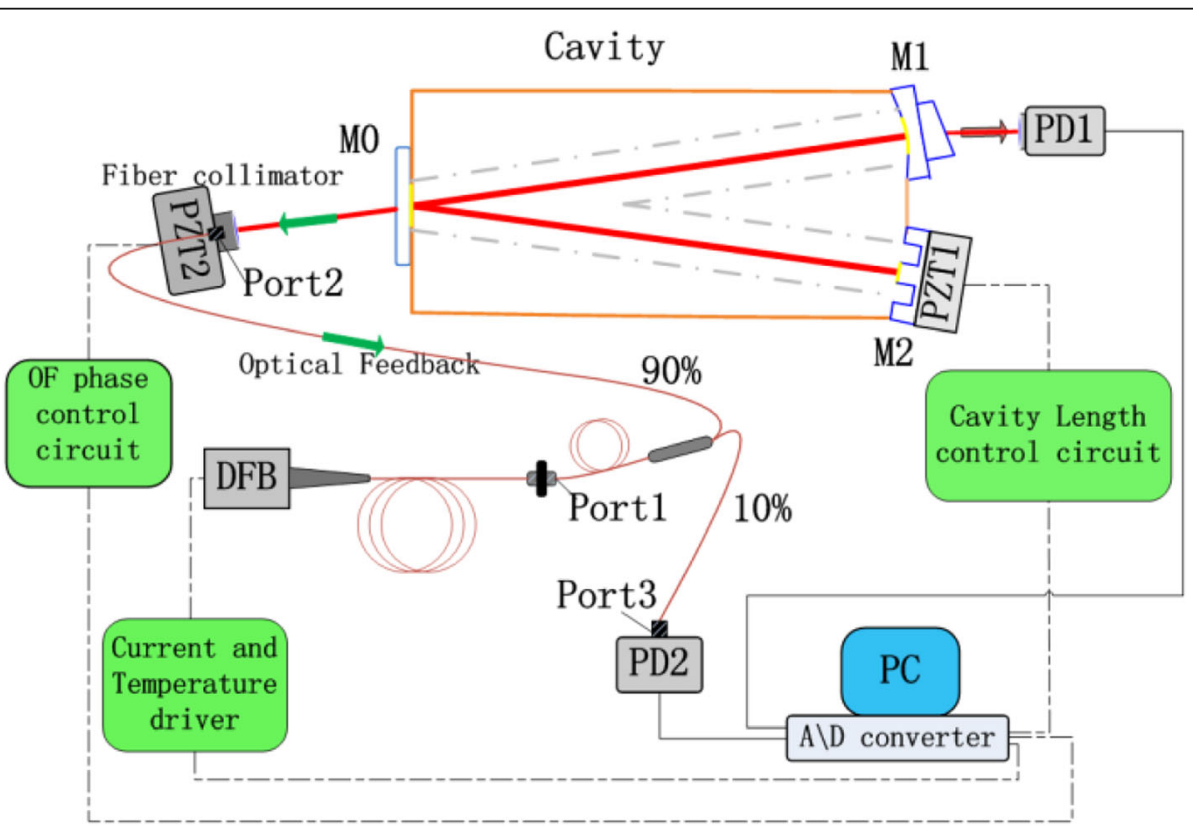

Fig. 1 Schematic diagram of the OF-CEAS experimental setup employing a V-shaped cavity and a fibered DFB laser diode. The direct reflection at MO will not return to the DFB, while part transmission light of the intra-cavity resonant field offers frequency selective optical feedback. The PZT2 mounted on the fiber collimator is used to modulate the phase of the feedback light, and the PZT1 mounted on M2 can adjust the cavity length to obtain cavity resonance 
As shown in Fig. 1, this V-shaped cavity can obtain the resonant optical feedback while avoiding the direct reflection at M0. In this experimental setup, the light from DFB laser diode is coupled into a singlemode polarization maintaining (PM) fiber, then the pigtail output is connected with the port1 of a PM fiber coupler (90:10, $1550 \mathrm{~nm}$ Center Wavelength), and the port 2 and port3 of this fiber coupler are connected with a fiber collimator (F240APC-1550, Thorlabs, Newton, NJ, USA) and a photo-detector (PDA400, Thorlabs, Newton, NJ, USA), respectively. The collimated light from the fiber collimator is then injected into the V-shaped cavity at M0. When the cavity resonance occurs, part of resonant light return to the laser diode, and PZT2 can actively optimize the feedback light phase versus the laser diode output. As we know, a small fraction of feedback resonant light can efficiently narrow the linewidth of the laser diode and also stabilize the laser frequency on the cavity's resonance frequency automatically [11]. This is extremely valuable to sensitively detect the narrow separated absorption lines of gas molecule. It should be noted that the fibered DFB laser diode used in this setup doesn't include an optical isolator which would prevent feedback light from the resonant cavity.

For traditional CEAS system, the spectral resolution is usually limited by the free spectral range (FSR) of the resonant cavity [12]. While in our setup, the PZT1 mounted on M2 can precisely modulate the cavity length to achieve the cavity's resonance for each fixed laser frequency, and the spectral resolution is mainly determined by frequency tuning resolution of the DFB diode laser. Therefore, the spectral resolution of this scheme can be efficiently improved to about $0.002 \mathrm{~cm}^{-1}$. Furthermore, in this setup, the optical feedback ratio can be regulated by applying replaceable PM fiber coupler which have different coupling ratio. For automatic operation, voltage signals of the photo-detectors are digitized with a 24-bit dynamic signal acquisition and generation device (NI-PCI-4461, $204.8 \mathrm{kS}$ / s, National Instrument, Austin, TX, USA). An analogic feedback circuit for PZT2 modulation is applied, which apply the cavity mode profile as presented in transmission signal during a laser scan to generate a real-time error signal. Signal processing and control signal generation are simultaneously achieved with self-compiled software based on LabVIEW. Finally, the fitting of absorption spectra based on HITRAN database and the calculation of gas concentration are obtained after each laser scan.

\section{Experimental phenomenon and theoretical analysis}

Our primary investigation is performed with residual air in the cavity, and a fibered $1531 \mathrm{~nm}$ DFB diode laser is used to match several absorption lines of $\mathrm{H}_{2} \mathrm{O}$ around $6530 \mathrm{~cm}^{-1}$. Owing to the high precision DFB diode laser driving system, high resolution of frequency tuning is attained. In the case of current tuning from 40 to $120 \mathrm{~mA}$, the range is about $2.75 \mathrm{~cm}^{-1}$ in the spectral range of $6528.95 \sim 6531.70 \mathrm{~cm}^{-1}$ by setting its operating temperature at $25^{\circ} \mathrm{C}$. In Fig. 2, the normalized voltage signals of PD1 is presented, it is obvious that this spectrum is a combination of a periodic signal and some absorption lines.

As we know, some periodic spectral signals existing in CRDS and CEAS have been observed and studied, including the spectral ripple effect and the etalon effect. The etalon effect existing in the FabryPerot etalon has been widely studied during the past decays [13, 14], it is caused by the multiple-beam interference at two reflecting surfaces of the FabryPerot etalon. The spectral ripple is mainly caused by the small mirror defects or dust particles of the folded mirror, and it has been studied by J. Morville [15] and Z. Q. Tan [16] in detail. Comparing the spectral ripple effect with the transmitted light intensity signal observed in Fig. 2, the period of this spectral signal is totally different from the spectral ripple's period. According to the theory of spectral ripple effect, the period of this signal indicates that the length of V-Shaped cavity is just about $12 \mathrm{~mm}$ if this periodic spectral fluctuation is caused by the spectral ripple effect. Obviously, this length is not coincident with the cavity length $L$. It seems that this periodic signal in Fig. 2 is much more similar with the etalon effect. Considering that M1 and M2 are not parallel surface mirrors, and a prism is optically glued on M1 to eliminate the potential etalon effect caused by M2, this phenomenon is most likely caused by the incident mirror (M0, the thickness $\mathrm{d}$ is $6 \mathrm{~mm}$ ). The incident mirror can be regarded as a Fabry-Perot cavity with a light incidence angle of $3.7^{\circ}$ (with the surface Light intensity reflectivity $\mathrm{R}_{0}=$ $3.32 \%$ and $R_{1} \approx 99.99 \%$ for $\lambda=1530 \mathrm{~nm}$ ). The incident light (shown in Fig. $3 \mathbf{a}$ as A) split into multiple beams (shown in Fig. 3a as A-1, A-2...) before coupling into the cavity, and the intra-cavity resonance light (shown in Fig. $3 \mathrm{~b}$ as $\mathrm{B}$ and Fig. 3c as $\mathrm{C}$ ) incident upon M0 with an incidence angle of $3.7^{\circ}$ from the opposite direction, and also split into multi beams by the two surfaces of M0. This reflecting process will generate an optical path difference between these beams.

Considering the theoretical output beam diameter of fiber collimator $(1.6 \mathrm{~mm})$ and the waist diameter of intra-cavity resonance light $(1.14 \mathrm{~mm})$, only the 1 st and 2nd level transmitting light are considered. Therefore, when these beams are coupled into the fiber with an 


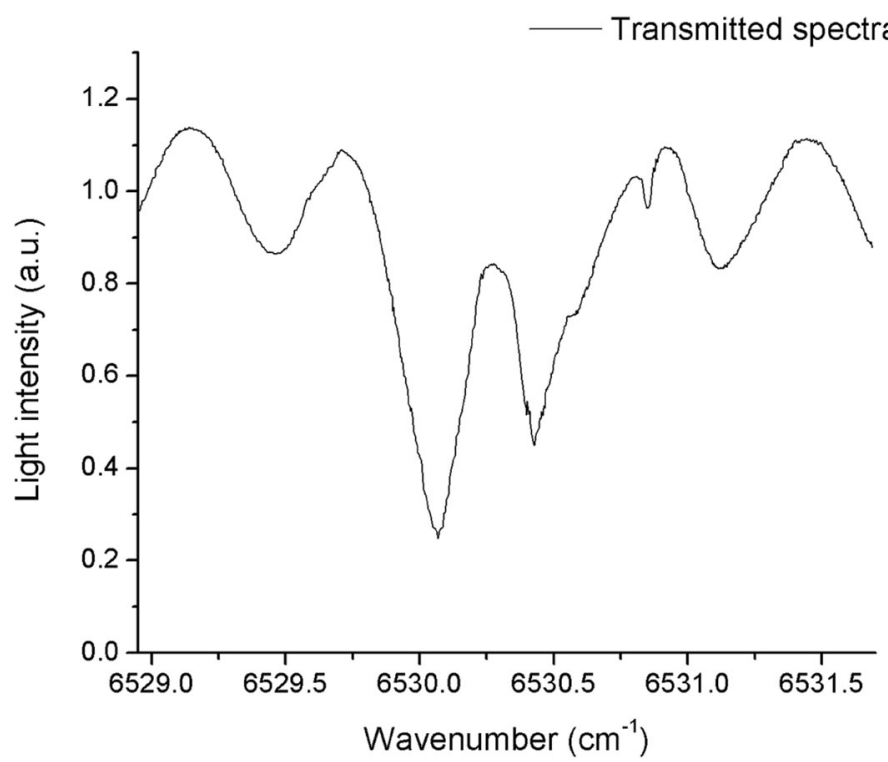

Fig. 2 The normalized OF-CEAS cavity transmission signal presenting a periodic spectral fluctuation include three strong absorption lines of molecular $\mathrm{H}_{2} \mathrm{O}$

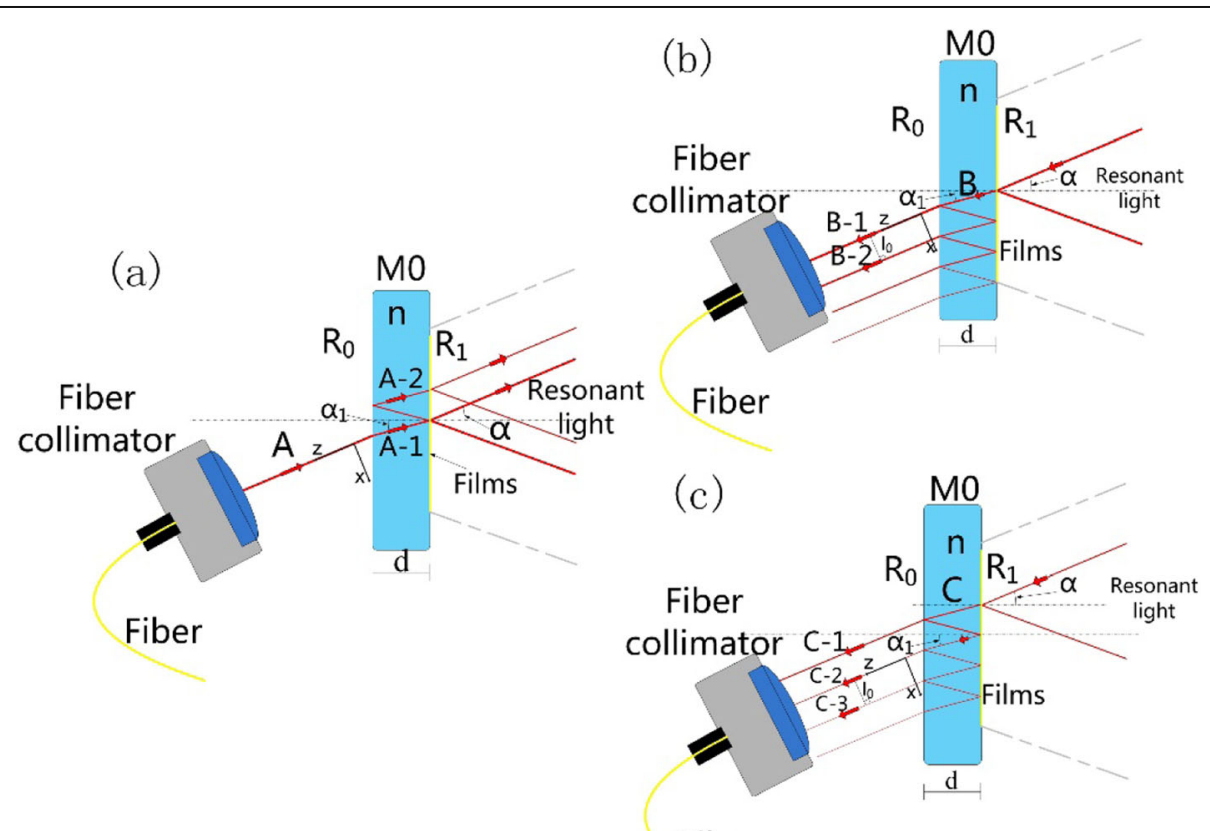

Fiber

Fig. 3 The incident mirror. In (a), the incident light A split into multiple beams, A-1 and A-2 can generate the intra-cavity resonance. $\mathbf{b}$ shows that the feedback light $B$ (which is produced by incident beam A-1) split into multiple beams before coupled into the fiber collimator; $\mathbf{c}$ shows that the feedback light $C$ (which is produced by incident beam A-2) split into multiple beams before coupled into the fiber collimator 
Anti-reflection (AR) coated aspheric lens, the multibeam interference phenomenon occurs in both the light intensity of feedback light and the cavity transmitting light. Considering the incident light $\mathrm{A}$ as:

$$
E_{i}(t)=A_{0} \cdot e^{-i(\omega t+k x)},(\omega=2 \pi v)
$$

Here we assume the resonance field of the Vshaped cavity to be fundamental $\mathrm{TEM}_{0,0}$ mode, and the laser beam is Gaussian. Due to the limitation of the fiber collimator (diameter of AR coated aspheric lens is $11 \mathrm{~mm}$ ), only qualified feedback light beam can be coupled into the fiber, distance between two adjacent transmitting beams is $l_{0}=2 d \cdot \cos \alpha \cdot \tan \alpha_{1}$. Since M1 and M2 are spherical mirrors with $2 \mathrm{~m}$ radius, the waist of the Gaussian beam is exactly on the incident mirror, and the radius of waist size is $\omega_{0}=$ $0.57 \mathrm{~mm}$. Here, by the integral operation, the total coupling light of the transmitting light field is calculated as:

$$
\begin{aligned}
E_{T}= & A_{0} \cdot \sqrt{\left(1-r_{0}^{2}\right)\left(1-r_{1}^{2}\right)\left(1-r_{2}^{2}\right)} \cdot e^{-i(\omega t+k x)} \\
& \cdot e^{-i\left(\delta_{0}+\delta_{1}\right)} \cdot\left(1+\phi_{0} r_{0} r_{1} e^{-i 2 \delta_{0}}\right) \cdot \phi
\end{aligned}
$$

The total coupling light of the feedback light field is:

$$
\begin{aligned}
& E_{F}=A_{0} \cdot\left(1-r_{0}^{2}\right)\left(1-r_{1}^{2}\right) r_{2} \cdot e^{-i(\omega t+k x)} \cdot e^{-i 2\left(\delta_{0}+\delta_{1}\right)} \\
& \cdot\left(1+\phi_{1} r_{0} r_{1} e^{-i 2 \delta_{0}}+\phi_{0} \phi_{2} r_{0} r_{1} e^{-i 2\left(\delta_{0}+\delta_{1}\right)}+\phi_{0} r_{0}^{2} r_{11}^{2} e^{-i 2\left(2 \delta_{0}+\delta_{1}\right)}\right) \cdot \phi
\end{aligned}
$$

Here $\quad \delta_{0}=n d \cdot \frac{2 \pi}{\lambda} / \cos (\alpha), \quad \delta_{1}=\delta_{2}=L \cdot \frac{2 \pi}{\lambda}, \quad \phi$ $=1 / 1-r_{1}^{2} r_{2} r_{3} e^{-i 2\left(\delta_{1}+\delta_{2}\right)}, n$ is the reflective index of quartz glass $(n=1.444), d$ is the thickness of M0 $(d=6 \mathrm{~mm}), L$ is the length of each cavity arm $(L=250 \mathrm{~mm})$, and $\alpha$ is the incidence angle $\left(\alpha=3.7^{\circ}\right) . r_{0}, r_{1}, r_{2}$, and $r_{3}$ represent the amplitude reflectivity of the mirror surface $\left(r_{0}^{2}=3.32 \%\right.$, $\left.r_{1}^{2}=r_{2}^{2}=r_{3}^{2} \approx 99.99 \%\right), \phi_{0}, \phi_{1}$ and $\phi_{2}$ are the coupling coefficient of incident beam A-2, B-2 and C-2, respectively ( $\phi_{0} \approx 80 \%, \phi_{1} \approx 80 \%, \phi_{2} \approx 100 \%$ ). The coupling coefficient is mainly determined by the optical path difference between these beams and the optical axis of cavity (or optical axis of output laser), as well as the diameter of laser beam.

To confirm this assumption, we did some adjustments with this experimental setup. The fiber coupler in Fig. 1 is replaced by a $2 \times 2 \mathrm{PM}$ Fiber Coupler (50: 50, $1550 \mathrm{~nm}$ Center Wavelength), and the feedback light is divided into two portions. One of them is connected to PD2 to observe the feedback light intensity, and another one returns to the DFB laser diode. According to our multi-beam interference assumption and simulation based on Formula (2) and (3), the interference phenomenon in feedback light intensity is strengthened for it passes through M0 twice. As shown in Figs. 4 and 5, the experimental results confirm to the theoretical simulation very well, obviously, the spectral fluctuation in the feedback light is more obvious than which in the transmitted light.

In Fig. 4, the black dots are the transmitted light intensity signal measured by PD1. The red dots show the calculated result based on Formula (2) and HITRAN 2012 database, which contain the periodic spectral fluctuation and several absorption lines of $\mathrm{H}_{2} \mathrm{O}$ around $6530 \mathrm{~cm}^{-1}$. The comparison of the measured spectra in Figs. 4 and 5 show that the amplitude of the periodic spectral fluctuation in feedback light signal is larger than which in transmitted light signal. It should be noted that the concentration of $\mathrm{H}_{2} \mathrm{O}$ in Figs. 4 and 5 has a preset value of $1.5 \%$, and the simulation results is revised after the effective value is calculated in Fig. 6.

Since that these experimental results agree well with the mathematical modeling results, the main cause of this periodic spectral fluctuation is determined to be the multi-beam interference of resonance light at the incident mirror (M0). Methods to eliminate or weaken this effect are proposed as follows:

1) The surface of $\mathrm{M} 0$ are wedged;

2) The surface of M0 is coated with anti-reflecting film;

3) A prism is optically glued on the backside of M0;

4) Increase the thickness of $\mathrm{M} 0$ (show as d).

In methods 1 and 3 , the non-parallel surfaces of M0 will eliminate the interference terms. With respect to method 2, the reflectivity $R_{O}$ is reduced when the surface is coated with anti-reflection film, and the interference terms will decrease as shown in Formula (2) and (3). In method 4, the distance between two adjacent transmitting beams is $l_{0}$, it is obvious that $l_{0}$ is proportional to $d$ (the thickness of M0). When $l_{0}$ is larger enough, the beam A-2 will not be resonant considering the limited area of film (diameter $\phi=10$ $\mathrm{mm}$ ), and the beam B-2 will not be coupled into the fiber because of the diameter of fiber collimator (diameter $\phi=11 \mathrm{~mm}$ ).

In all these methods mentioned above, all the interference terms can be totally eliminated with methods 1 and 3. However, the observed periodic spectral fluctuation in this fibered OF-CEAS setup does not appear in typical "open light path" OF-CEAS system, the usage of a prism makes our experimental setup more flexible. In general, we think "A prism is optically glued on the backside of M0" is the most simple and practical method for our experimental setup. To verify its effectiveness, a right angle prism with a 


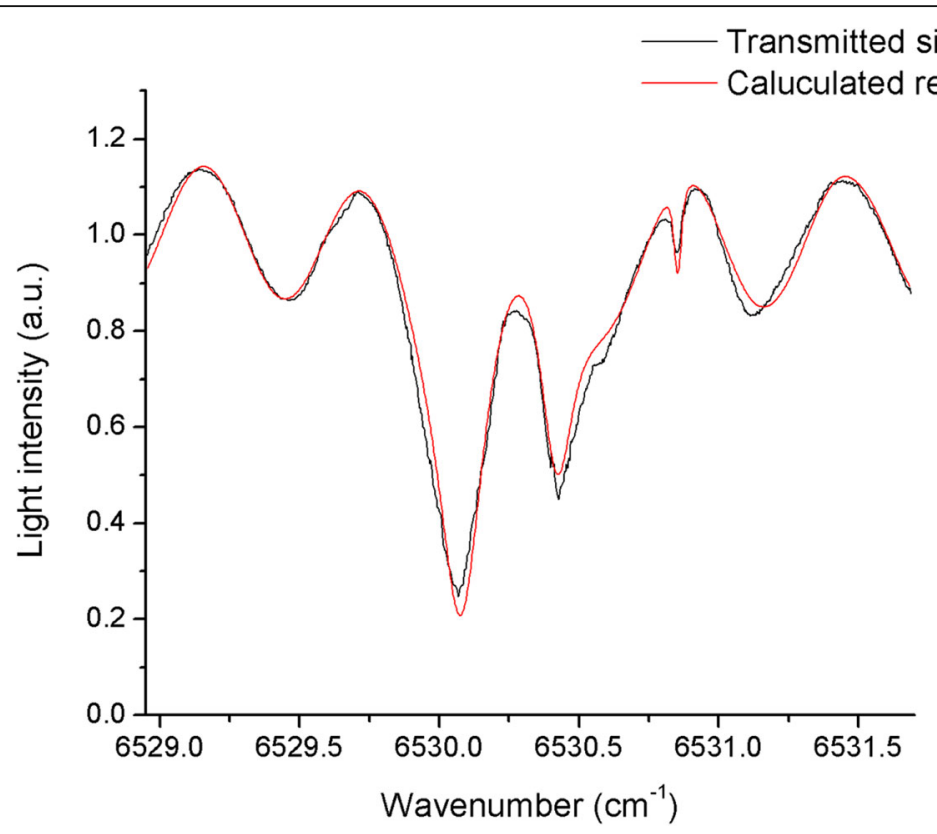

Fig. 4 The cavity transmitted light signal (black) and the simulation results (red). The simulation results include the interference effect calculated using the Formula (2) and simulation of the absorptions based on HITRAN $2012\left(P=1.0 \mathrm{~atm}, \mathrm{~T}=25^{\circ} \mathrm{C}\right)$

sharp angle of 27 degrees is optically glued on the backside of M0. The OF-CEAS signal is measured again with the same measurement subject, and the result is shown in Fig. 6a. Apparently, the periodic spectral fluctuation disappears, and this experimental result coincides with the analyses described above.
Figure 6 shows a single laser scan of our OF-CEAS system in about $10 \mathrm{~s}$. The $1531 \mathrm{~nm}$ DFB diode laser is tuned to match several absorption lines of $\mathrm{H}_{2} \mathrm{O}$ around 6530 $\mathrm{cm}^{-1}$ (the strongest absorption line intensity is $2.926 \times 10^{-}$ ${ }^{24} \mathrm{~cm}^{-1} /\left(\right.$ molecule $\left.\times \mathrm{cm}^{-2}\right)$ at $6530.05 \mathrm{~cm}^{-1}$ according to HITRAN database). The absorption coefficient $\alpha$ is

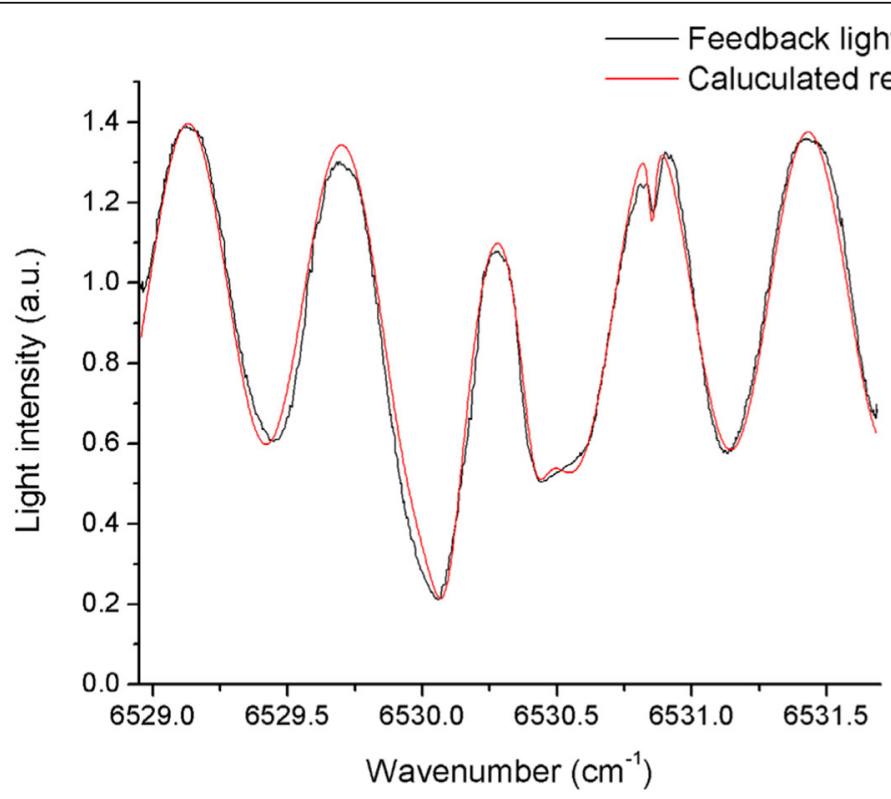

Fig. 5 The feedback light signal (black) and the simulation results (red). The simulation results includes the interference effect calculated using the Formula (3) and simulation of the absorptions based on HITRAN $2012\left(P=1.0 \mathrm{~atm}, \mathrm{~T}=25^{\circ} \mathrm{C}\right)$ 


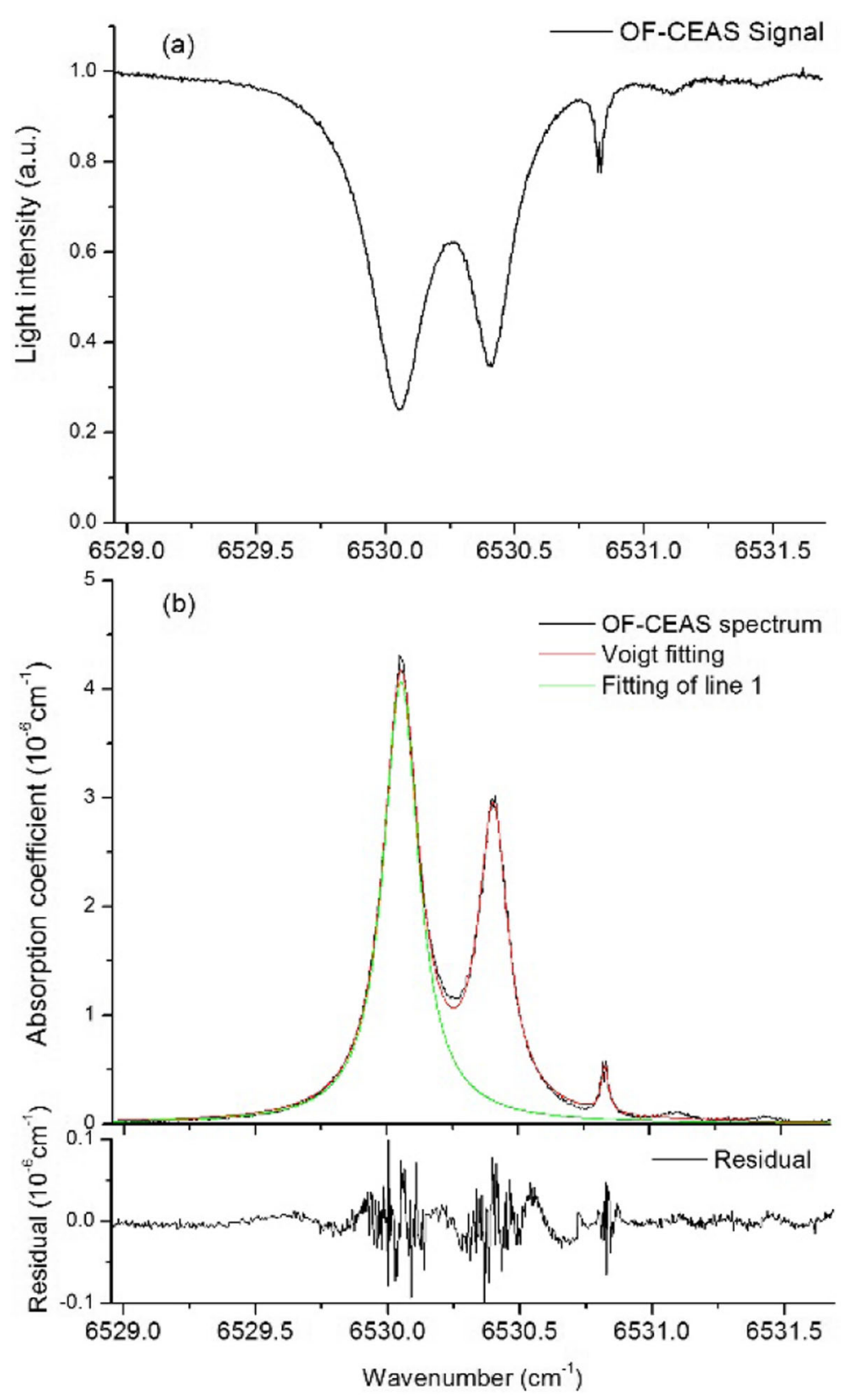

Fig. $\mathbf{6}$ a shows the transmitted light signal of the developed OF-CEAS system; b shows the absorption coefficient spectrum (black) and Voigt fitting of the absorptions of molecular $\mathrm{H}_{2} \mathrm{O}$ (red and green) based on HITRAN $2012\left(\mathrm{P}=1.0 \mathrm{~atm}, \mathrm{~T}=25^{\circ} \mathrm{C}\right.$ ); the bottom shows the fit residual

determined by the transmitted intensity (I) with the OFCEAS formula [17]:

$$
\alpha=\frac{1-R_{e f f}}{L_{0}}\left(\sqrt{\frac{I_{0}}{I}}-1\right)
$$

where $L_{O}$ is the physical cavity length, $R_{e f f}$ is the equivalent reflectivity of mirrors which is determined by the "empty cavity" ring-down time $\left(\tau_{0}\right)$, therefore, $\left(1-R_{\text {eff }}\right)$ represents the cavity optical loss in a single pass. The reference intensity signal $\left(I_{0}\right)$ was confirmed by the transmitted light intensity without absorption.

The ultra-low loss coating is designed and manufactured by ourselves $(R \approx 99.99 \%$ at $1530 \pm 30 \mathrm{~nm})$. In this experimental setup, a fast fibered optical switch can be placed between the DFB laser diode and the fiber coupler to produce instantaneous interruption of the laser (10 ns). When the cavity is filled with pure $\mathrm{N}_{2}$, the "empty cavity" ring-down time $\tau_{0}$ is determined to be $\tau_{0}=7.75 \mu \mathrm{s}$ at $1531.4 \mathrm{~nm}$, corresponding to a cavity loss 
of $\left(1-R_{\text {eff }}\right)=215 \mathrm{ppm}$ in a single pass. For each mirror, the effective value of light intensity reflectivity is determined to be $99.98925 \%$, the approximate value of 99.99\% is used during the simulation of Formula (2) and (3). The residuals of the fitting indicate that the $1 \sigma$ noise equivalent absorption coefficient of $7.6 \times 10^{-10} \mathrm{~cm}^{-1}$ $\mathrm{Hz}^{-1 / 2}$ is achieved, corresponding to a minimum detectable absorption coefficient of $\alpha_{\min }=3.4 \times 10^{-9} \mathrm{~cm}^{-1}$. Using the relation $\alpha=\mathrm{n}_{*} \sigma$ ( $\sigma$ represents the absorption cross-section for a predetermined gas) [18], the concentration of $\mathrm{H}_{2} \mathrm{O}$ in the cavity is calculated as $1.46 \%$. The higher performance can be obtained by the fitting of repeated absorption coefficient spectra, and a better minimum detectable absorption coefficient of $\alpha_{\min }=1.1 \times$ $10^{-9} \mathrm{~cm}^{-1}$ is achieved after the averaging of 10 laser scans.

\section{Conclusions}

We have presented a novel fibered OF-CEAS system with an enhanced spectral resolution. This OF-CEAS system require fewer optical components, it is suitable to the achievement of robust, low power, compact, and economical instruments for trace gas detection. For the periodic spectral fluctuation appearing in the primary investigation of this OF-CEAS system, its mechanism is analyzed and the mathematical model is proposed, and the experimental results agree well with the theoretical simulation. Some technical proposals are proposed to eliminate this periodic spectral fluctuation, and the experimental results show that this spectral fluctuation can be eliminated efficiently when a prism is optically glued on the backside of the incident mirror. With these improvement, the $1 \sigma$ noise equivalent absorption coefficient of $7.6 \times 10^{-10}$ $\mathrm{cm}^{-1} \mathrm{~Hz}^{-1 / 2}$ is achieved in a single scan within $10 \mathrm{~s}$, and real-time measurement of some trace gas at the ppb level can be achieved. The sensor based on this fibered OF-CEAS is compact, robust and economically available, it can offer accurate selectivity, high sensitivity, non-invasive and real time monitoring as well as no harm for patients when it is applied in human breath diagnosis. The next step of our work is exploiting the performance of this fibered OF-CEAS in the measurement of ammonia and hydrogen cyanide existing in exhaled human breath gas, the detection limit of this system would permit a concentration detection limit of $12.5 \mathrm{ppb}$ for $\mathrm{NH}_{3}$ at $1531 \mathrm{~nm}$. Furthermore, this fibered OF-CEAS setup permits easy replacement for the DFB laser operating in the range of cavity mirrors, the multi-component analysis of gas can be accomplished with this OF-CEAS setup. We are looking forward to developing an instrument for breath gas analysis which is suitable for clinical application.

\section{Acknowledgements}

Not applicable.

\begin{abstract}
Authors' contributions
$\mathrm{XL}$ proposed the concept of this study, and was a contributor in review and editing for this manuscript. ZT acquired the funding for this research work. $\mathrm{ZL}$ performed the investigation and experimental research, and was the major contributor in writing and editing the manuscript. All authors read and approved the final manuscript.
\end{abstract}

\section{Funding}

This research was funded by the Research Project of National University of Defense Technology (No. ZK16-03-21).

\section{Availability of data and materials}

The datasets used and analyzed during the current study are available from the corresponding author on reasonable request.

\section{Competing interests}

The authors declare that they have no competing interests.

Received: 26 July 2019 Accepted: 4 October 2019

Published online: 29 October 2019

\section{References}

1. Wojtas, J., Mikolajczyk, J., Nowakowski, M., Bielecki, Z:: Applying CEAS method to UV, VIS, and IR spectroscopy sensors. Opto. Electron. Rev. 59, 4 (2011)

2. Gianella, M., Ritchie, G.A.D.: Cavity-enhanced near-infrared laser absorption spectrometer for the measurement of acetonitrile in breath. Anal. Chem. 87, 6881-6889 (2015)

3. Ulanowska, A., Ligor, T., Michel, M., Buszewski, B.: Hyphenated and unconventional methods for searching volatile cancer biomarkers. Ecol. Chem. Eng. 17, 9-23 (2010)

4. Hodgkinson, H., Tatam, R.P.: Optical gas sensing: a review. Meas. Sci. Technol. 24, 15-17 (2013)

5. Desbois, T., Ventrillard, I., Romanini, D.: Simultaneous cavity-enhanced and cavity ringdown absorption spectroscopy using optical feedback. Appl. Phys. B Lasers Opt. 116, 465-201 (2004)

6. Mottoros, V., Morville, J., Rairoux, P.: Mode-by-mode optical feedback: cavity ringdown spectroscopy. Appl. Phys. B Lasers Opt. 87, 531-538 (2007)

7. Durand, M., Morville, J.: Shot-noise-limited measurement of sub-parts-pertrillion birefringence phase shift in a high-finesse cavity. Phys. Rev. A. 82, 031803 (2010)

8. Bergin, A.G.V., Hancock, G., Ritchie, G.A.D., Weidmann, D.: Linear cavity optical-feedback cavity-enhanced absorption spectroscopy with a quantum cascade laser. Opt. Lett. 38, 14 (2013)

9. Courtillot, I., Morville, J., Ros-Motto, V., Romanini, D.: Sub-ppb $\mathrm{NO}_{2}$ detection by optical feedback cavity-enhanced absorption spectroscopy with a blue diode laser. Appl. Phys. B Lasers Opt. 85, 407-412 (2006)

10. Engeln, R., Berden, G., Peeters, R., Meijer, G.: Cavity enhanced absorption and cavity enhanced magnetic rotation spectroscopy. Rev. Sci. Instrum. 69, 3763 (1998)

11. Dahmani, B., Hollberg, L., Drullinger, R.: Frequency stabilization of semiconductor lasers by resonant optical feedback. Opt. Lett. 11, 876-878 (1987)

12. Morville, J., Kassi, S., Chenevier, M.: Fast, low-noise, mode-by-mode, cavityenhanced absorption spectroscopy by diode-laser self-locking. Appl. Phys. B Lasers Opt. 80, 1027-1038 (2005)

13. Hosaka, K., Inaba, H., Akamatsu, D., Yasuda, M., Sugawara, J., Onae, A., Hong, F.L.: A Fabry-Perot etalon with an ultralow expansion ceramic spacer. Jpn. J. Appl. Phys. 52, 032402 (2013)

14. Lumeau, J., Glebov, L.B., Smirnov, V.: Tunable narrowband filter based on a combination of Fabry-Perot etalon and volume Bragg grating. Opt. Let. 31, 16 (2006)

15. Morville, J., Romanini, D., Kachanov, A.A., Chenevier, M.: Two schemes for trace detection using cavity ringdown spectroscopy. Appl. Phys. B Lasers Opt. 78, 465-476 (2004)

16. Tan, Z.Q., Luo, Z.F., Liu, S.Q., Zhang, X.B., Long, X.W.: Further study of spectral ripple with a laser scattering measurement apparatus. Opt. Commun. 393, 103-106 (2017) 
17. Katherine, M., Manfred, J., Kirkbride, M.R., Ciaffoni, L., Peverall, R., Ritchie, G.A. D.: Enhancing the sensitivity of mid-IR quantum cascade laser-based cavityenhanced absorption spectroscopy using RF current perturbation. Opt. Lett. 39, 24 (2014)

18. Gherman, T., Eslami, E., Romanini, D.: High sensitivity broad-band modelocked cavity-enhanced absorption spectroscopy: measurement of $\operatorname{Ar}^{*}(3 \mathrm{P} 2)$ atom and ion densities. J. Phys. D. Appl. Phys. 37, 17 (2004)

\section{Publisher's Note}

Springer Nature remains neutral with regard to jurisdictional claims in published maps and institutional affiliations.

Submit your manuscript to a SpringerOpen ${ }^{\odot}$ journal and benefit from:

- Convenient online submission

- Rigorous peer review

- Open access: articles freely available online

- High visibility within the field

- Retaining the copyright to your article

Submit your next manuscript at $\boldsymbol{\wedge}$ springeropen.com 